\title{
Does Patella Tendon Tenodesis Improve Tibial Tubercle Distalization in Treating Patella Alta? A Computational Study
}

\author{
Li Yin MD, PhD, Tzu-Chieh Liao MS, PT, Liu Yang MD, PhD, \\ Christopher M. Powers PT, PhD, FACSM, FAPTA
}

Received: 9 February 2016/Accepted: 5 August 2016/Published online: 30 August 2016

(C) The Association of Bone and Joint Surgeons (B) 2016

\begin{abstract}
Background Patellofemoral malalignment associated with patella alta may cause pain and arthritis; because of this, the condition sometimes is treated surgically. Two common procedures are tibial tubercle distalization with or without patellar tendon tenodesis. However, the biomechanical consequences of these interventions for patella alta are not clearly understood.

Questions/purposes We evaluated changes in patellofemoral joint contact mechanics after tibial tubercle

Each author certifies that he or she, or a member of his or her immediate family, has no funding or commercial associations (eg, consultancies, stock ownership, equity interest, patent/licensing arrangements, etc) that might pose a conflict of interest in connection with the submitted article.

All ICMJE Conflict of Interest Forms for authors and Clinical Orthopaedics and Related Research ${ }^{\circledR}$ editors and board members are on file with the publication and can be viewed on request.

Clinical Orthopaedics and Related Research ${ }^{\mathbb{R}}$ neither advocates nor endorses the use of any treatment, drug, or device. Readers are encouraged to always seek additional information, including FDAapproval status, of any drug or device prior to clinical use.

Each author certifies that his or her institution approved the human protocol for this investigation, that all investigations were conducted in conformity with ethical principles of research, and that informed consent for participation in the study was obtained.

This work was performed at the University of Southern California, Los Angeles, CA, USA.
\end{abstract}

L. Yin, L. Yang

Center for Joint Surgery, Southwest Hospital, The Third Military

Medical University, Chongqing, People's Republic of China

L. Yin, T.-C. Liao, C. M. Powers $(\bowtie)$

Jacquelin Perry Musculoskeletal Biomechanics Research Lab, Division of Biokinesiology and Physical Therapy, University of Southern California, 1540 E Alcazar Street, CHP-155,

Los Angeles, CA 90089-9006, USA

e-mail: powers@usc.edu distalization and tibial tubercle distalization combined with patella tendon tenodesis. Specifically, we asked: (1) Are there biomechanical differences between these two types of procedures? (2) Is there an ideal range to distalize the patella? Methods Subject-specific finite-element models were created for 10 individuals with patella alta (mean InsallSalvati ratio of $1.34 \pm 0.05$ ). Input parameters for the finite-element models included subject-specific joint geometry, quadriceps muscle forces, and weightbearing patellofemoral joint kinematics. Virtual operations were conducted to simulate the two procedures. For distalization, the tibial tubercle and patella were displaced distally $4 \mathrm{~mm}$ to $20 \mathrm{~mm}$ in 4-mm increments based on the original model. At each level of distalization, the patella tendon was attached back to its original insertion to simulate the additional tenodesis procedure. Cartilage stress, contact area, and contact forces were quantified and compared between procedures and distalization levels.

Results Distalization and distalization + tenodesis reduced patellofemoral joint stress compared with the baseline of $1.02 \pm 0.11 \mathrm{MPa}$. Distalization led to lower cartilage stress than distalization + tenodesis, and the effect size was relatively large $(0.88 \pm 0.10 \mathrm{MPa}$ vs $0.92 \pm 0.10 \mathrm{MPa}$; mean difference, $0.04 \mathrm{MPa}\left[\begin{array}{lll}95 \% & \mathrm{CI} & 0.02 \mathrm{MPa}-0.05 \mathrm{MPa}\end{array}\right]$, $\mathrm{p}<0.01$; effect size of 1.64 [Cohen's d], with Insall-Salvati ratio decreased to 0.95 ). For both procedures, the trend of stress reduction plateaued when the Install-Salvati ratio approached 0.95 .

Conclusions Cartilage stress appears lower using distalization as opposed to distalization + tenodesis in this finite-element analysis simulation. An Insall-Salvati ratio of 0.95 may be an ideal level for distalization; further distalization does not show additional benefits.

Clinical Relevance This study suggests that distalization may result in less stress than distalization + tenodesis, 
therefore future clinical research might be preferentially directed toward evaluating isolated distalization procedures.

\section{Introduction}

The Insall-Salvati ratio is the length of the patellar tendon divided by the proximal-to-distal length of the patella, measured on lateral radiographs of the knee; patella alta, which is defined by an Insall-Salvati ratio greater than 1.2, is a common anatomic abnormality of the patellofemoral joint [10]. Patella alta has been reported to be associated with patella subluxation, episodic dislocation, and patellofemoral joint instability $[18,31]$. Patellofemoral malalignment associated with patella alta is believed to cause excessive wear of the cartilage, contributing to the development of patellofemoral pain and arthritis $[13,15,27]$. Biomechanical studies have shown that patella alta can result in decreased patellofemoral joint contact area and elevated contact stresses [26, 30, 32].

A common surgical procedure for patella alta is tibial tubercle distalization (referred to as distalization hereafter) [18]. By distally transferring the insertion of the patella tendon, the patella can be repositioned to an appropriate height in the trochlea, thus improving patellar stability (Fig. 1). Neyret et al. [21] has suggested the patella tendon tenodesis be performed in addition to the tibial tubercle distalization (referred to as distalization + tenodesis hereafter) to restore the normal length of patella tendon and provide further patella stability (Fig. 1). Clinically, studies have shown that both procedures decrease patellar height, reduce the chance of patella dislocation, and result in improved patient-reported outcomes [18-20, 24].

Although distalization and distalization + tenodesis have been shown to relieve pain and improve patella stability [18-20, 24], the underlying biomechanical changes associated with these two surgical procedures are not well understood. Specifically, changes in joint contact forces, contact area, and cartilage stress as a function of the magnitude of patella distalization remain unclear.

Using a finite-element analysis approach, we therefore sought to quantify the biomechanical changes associated with distalization and distalization + tenodesis. In addition, we sought to address the following questions: (1) Are there biomechanical differences between these two types of procedures? (2) Is there an ideal range to distalize the tibial tubercle?

\section{Patients and Methods}

The subjects included in this study were selected from an existing model database of the patellofemoral joint in our laboratory. This database contains subject-specific finiteelement models of women with a diagnosis of patellofemoral pain and healthy control subjects matched by age and sex. The previously established exclusion criterion used for the database included no history of knee surgery, no history of traumatic patella dislocation, and no evidence of cartilage disorders. All subjects previously had provided informed consent approved by the Health Sciences Campus of the University of Southern California.

Based on the procedures described, MR images were evaluated to identify individuals in the database with patella alta. Using the criteria of an Insall-Salvati ratio greater than 1.2, 10 subjects were identified. The sample size was estimated referring to similar studies [7, 8, 25], and because this was the number of female subjects we had available in our database. The mean age, height, body weight, and Insall-Salvati ratio of these individuals was
Fig. 1A-C The schematics show (A) patella alta, (B) the procedure of tibial tubercle distalization and $(\mathbf{C})$ the procedure of tibial tubercle distalization plus patella tendon tenodesis. For tibial tubercle distalization, the tibial tubercle is osteotomized as a bone block and then fixed in a distalized position. For patella tendon tenodesis, the patella tendon is sutured back to the position of its original insertion, in addition to tibial tubercle distalization.
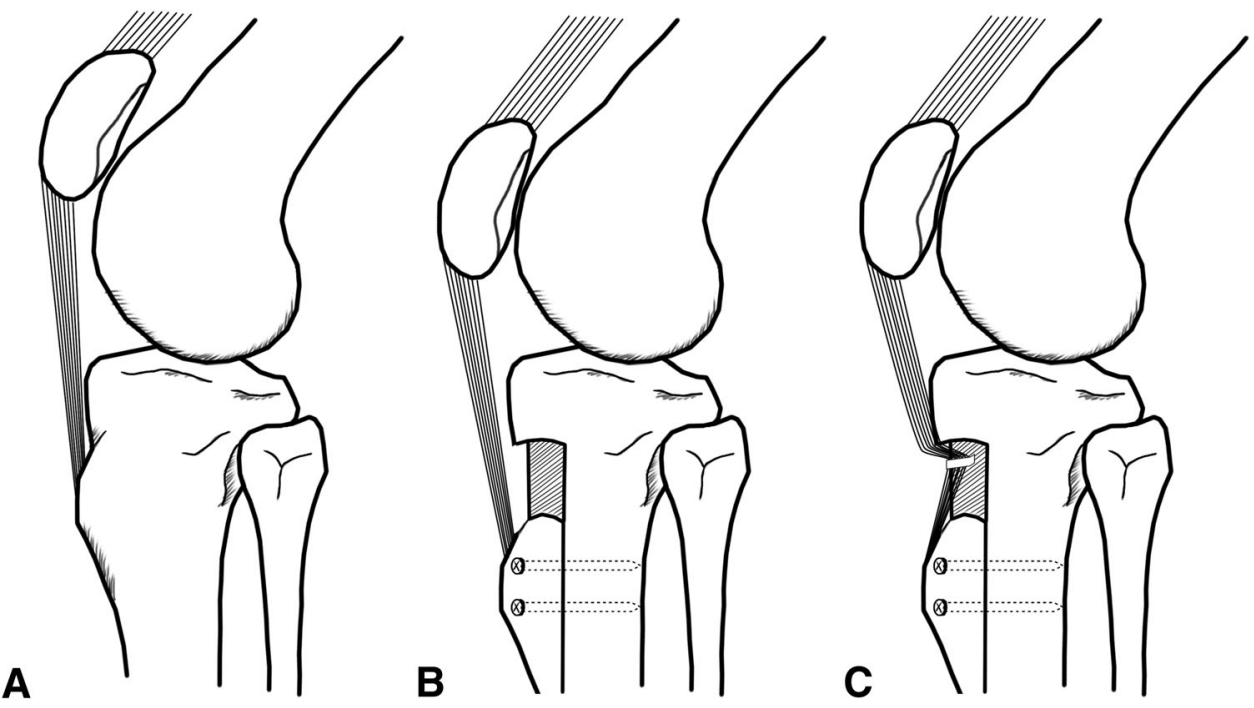
$28 \pm 4$ years, $1.7 \pm 0.1 \mathrm{~m}, 63 \pm 8 \mathrm{~kg}$, and $1.34 \pm 0.05$, respectively. Of the 10 subjects selected, seven had symptomatic patellofemoral pain (retropatellar pain during daily activities, such as squatting, chair climbing, isometric quadriceps contraction), whereas the remaining three were pain-free.

\section{MR Imaging and Patella Height Assessment}

Each subject underwent three stages of imaging using a 3.0-T MR scanner (General Electric Healthcare, Milwaukee, WI, USA). First, high-resolution images (threedimensional [3-D], fast Spoiled Gradient Recalled Echo [SPGR] sequence; repetition time, $14.5 \mathrm{~ms}$; echo time, $2.8 \mathrm{~ms}$; flip angle, 10 ; matrix, $320 \times 320$; field of view, $16 \mathrm{~cm}$; slice thickness, $1.0 \mathrm{~mm}$ ) were acquired with the knee fully extended. A second imaging protocol (3-D, fast SPGR sequence; repetition time, $14.3 \mathrm{~ms}$; echo time, $3.6 \mathrm{~ms}$; flip angle, 10; matrix, $320 \times 160$; field of view, $16 \mathrm{~cm}$; slice thickness, $2.0 \mathrm{~mm}$ ) then was performed at $45^{\circ}$ knee flexion with weightbearing forces equivalent to $25 \%$ body weight applied to the scanned lower limb using a MR-compatible loading apparatus. These images were used to register the geometric models built from the highresolution images, thus representing their weightbearing positions. Finally, high-resolution images of the thigh in the sagittal, axial, and coronal planes (3-D SPGR sequence; repetition time, $9.4 \mathrm{~ms}$; echo time, $4.1 \mathrm{~ms}$; flip angle, 20; matrix, $384 \times 384$; field of view, $46 \mathrm{~cm}$; slice thickness, $2 \mathrm{~mm}$ ) then were acquired. These images were used to estimate the 3-D fiber orientation and the cross-sectional area of each of the quadriceps muscles [9].

The Insall-Salvati ratio of each subject was quantified based on the methods described by Lee et al. [16]. Using midsagittal images, patella length was measured from the tip of the superior articular margin to the distal tip of the patella. Patella tendon length was measured from the distal tip of the patella to the tibial insertion at a point where the tibial tuberosity was most prominent. The Insall-Salvati ratio then was calculated as the length of the patella tendon divided by the length of the patella.

\section{Biomechanical Testing}

Subjects were instrumented for kinematic and EMG analysis as described in previous studies $[9,12,30]$. After a standing calibration trial, subjects were asked to hold a bilateral squat position at $45^{\circ}$ knee flexion with the trunk-maintained upright. Kinematic data were obtained using an 11-camera QUALISYS motion analysis system at $60 \mathrm{~Hz}$ (Qualisys Inc, Gothenburg, Sweden). Ground reaction forces were recorded at $1560 \mathrm{~Hz}$ using two force plates (Model OR6-6-1; AMTI, Watertown, MA, USA). EMG signals of selected knee musculature were recorded at $1560 \mathrm{~Hz}$ using preamplified, bipolar, surface electrodes (Motion Lab Systems, Baton Rouge, LA, USA). Kinematic, kinetic, and EMG data were recorded simultaneously. This information was used for estimation of quadriceps muscle forces, which was required as an input variable in the finite-element models (see subsequently for details).

\section{Individual Input Variables}

The input variables for the subject-specific finite-element models included joint geometry, joint kinematics, and quadriceps muscle forces (Fig. 2), acquired following procedures established in previous studies [9, 12]. Briefly, individual subject joint geometry was obtained from the high-resolution MR images using a commercial software package (sliceOmatic ${ }^{\mathrm{TM}}$; TomoVision ${ }^{\mathrm{TM}}$, Magog, Canada). Geometric models were registered manually to the weightbearing MR images. The 3-D force vector for each of the quadriceps muscles (rectus femoris, vastus intermedius, vastus medialis, and vastus lateralis) was estimated from the axial MR images of the thigh. Individual muscle-force magnitudes were estimated using a musculoskeletal modeling package (SIMM; MusculoGraphics Inc, Santa Rosa, CA, USA) based on subject-specific biomechanical data (kinematics, kinetics, EMG).

\section{Finite-element Modeling}

The preprocessing of finite-element models was performed in Hypermesh (http://www.altairhyperworks.com/product/ HyperMesh; Altair Engineering Inc, Troy, MI, USA). Geometric models of the femur, patella, tibia, and articular cartilage were assembled after being registered to their weight bearing positions (Fig. 3). Bony structures were defined as rigid bodies. The articular cartilage of the femur and patella was modeled using homogeneous isotropic tetrahedral continuum elements with an elastic modulus of 4.0 MPa [6] and a Poisson ratio of 0.47 [3]. The element size was set to $0.75 \mathrm{~mm}$ based on a previous mesh convergence analysis, which showed that a smaller element size did not result in meaningful change in the results but increased the computational time [9].

The patella tendon was modeled as six uniaxial tensileonly linear connectors with a total stiffness of $4334 \mathrm{~N} / \mathrm{mm}$. Patellofemoral joint contact was simulated with a surface coefficient of friction of 0.02 between cartilage [3,9]. Because the soft tissues controlling the rotation of the patellofemoral joint were not included in the models (such 
Fig. 2 The individual input variables used for the subjectspecific finite-element modeling of the patellofemoral joint are shown.

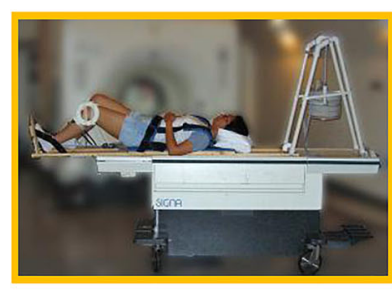

\section{Kinematics}

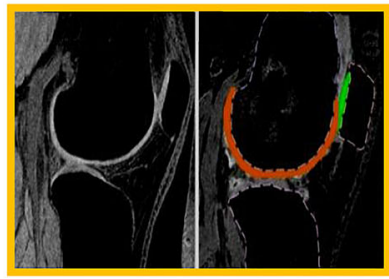

Geometry
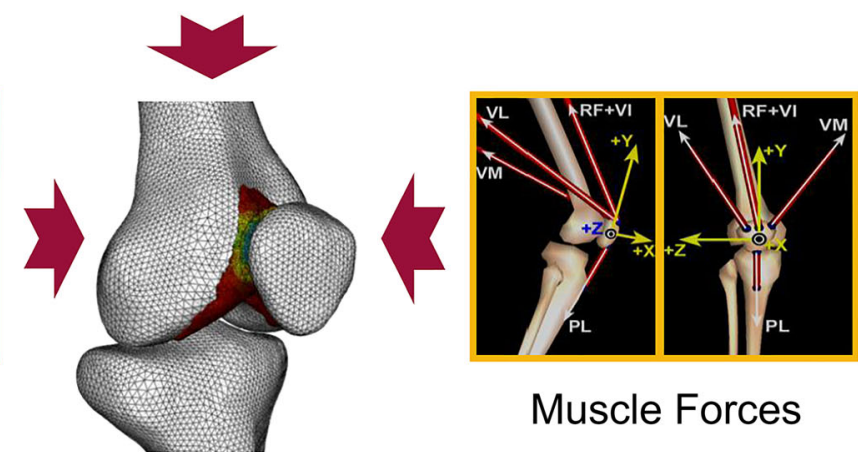

Muscle Forces

Finite Element Model

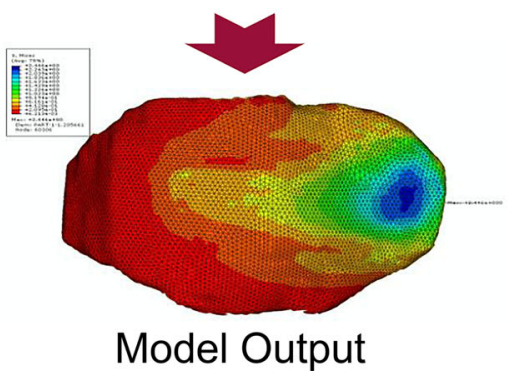

as ligaments and peripatellar retinaculum), the rotational degrees of freedom of the patella were constrained in the frontal and transverse planes. Rotation in the sagittal plane was permitted to allow the patella the ability to accommodate the varying patella heights induced by the simulated surgical procedures described subsequently. Model simulations were performed using Abaqus software (Version 6.12; Dassault Systèmes Simulia ${ }^{\circledR}$ Corp, Johnston, RI, USA). The modeling approach described previously was validated in a previous study by comparing the model-predicted contact area and patella alignment with actual values as measured on weightbearing MR images, showing average deviations of $3.0 \%$ and $2.7 \%$, respectively [9].

Simulation of Distalization and Distalization + Tenodesis

The two surgical procedures were simulated by repositioning the patella and the tibial tubercle based on the original models. Models for distalization were created by inferiorly translating the tibial tubercle along the longitudinal axis of the tibia (Fig. 4). Similarly, the patella was displaced distally the same distance so that the length of the patella tendon could be maintained. Multiple models were generated using various distances of distalization, ranging from $4 \mathrm{~mm}$ to $20 \mathrm{~mm}$ in $4-\mathrm{mm}$ increments.

The distalization models were further modified to simulate distalization + tenodesis. This was done by restoring the patella tendon insertion to its original position after distalization of the patella (thus resulting in a shortened patella tendon; Fig. 4). The Insall-Salvati ratio was reevaluated in each derived model. For distalization and distalization + tenodesis, the length of the patella tendon was quantified from the distal pole of the repositioned patella to the site of the original patella tendon insertion. The mean Insall-Salvati ratio of all subjects before model manipulation was $1.34 \pm 0.05$. On average, distalization of the tibial tubercle by $4 \mathrm{~mm}$ decreased the Insall-Salvati ratio by 0.1 . In addition, the maximum amount of distalization evaluated $(20 \mathrm{~mm})$ reduced the mean Insall-Salvati ratio to $0.85 \pm 0.07$. 


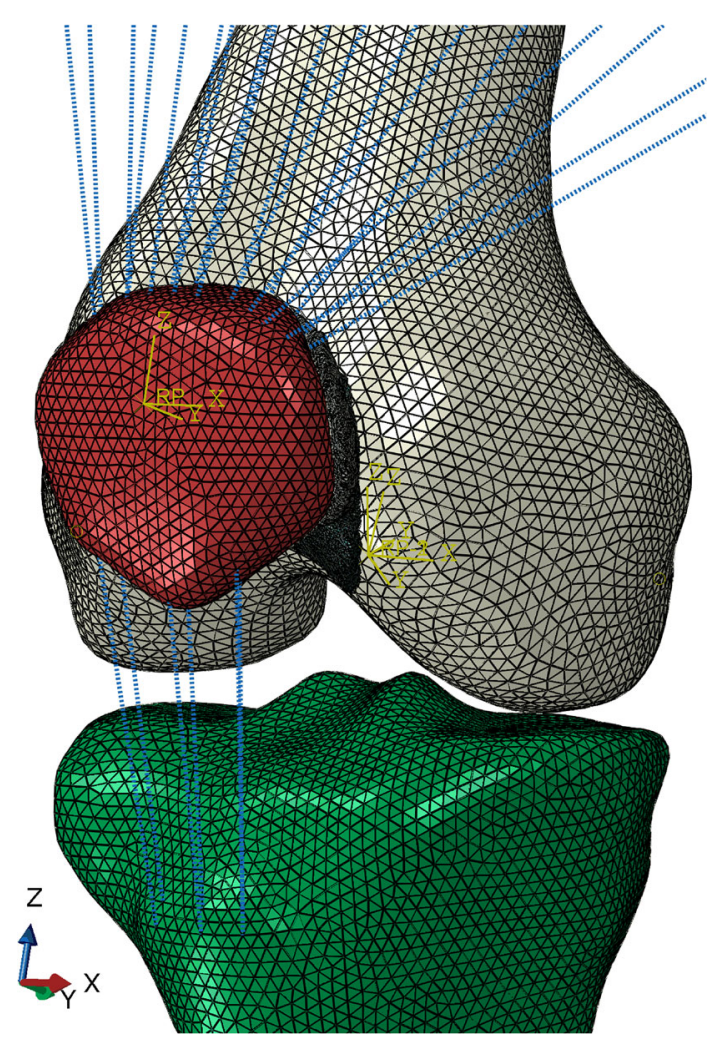

Fig. 3 A representative finite-element model of the patellofemoral joint is shown. The arrows at the lower left corner and in the model represent the global coordinate system and the local coordinate systems for bone parts, with $\mathrm{X}, \mathrm{Y}$ and $\mathrm{Z}$ axes defined.
Given that the changes in patella position would influence the moment arm of the quadriceps muscle group, muscle forces were scaled such that each model simulation had the same knee extension moment to that of the original model. The extensor moment arm was calculated based on the flexion-extension axis of the knee, which was estimated by finding the line connecting the centers of the posterior femoral condyles. As such, models for each subject had varied muscle force inputs but an identical knee moment output.

\section{Data Analysis and Statistics}

Three parameters were output from finite-element models to quantify contact mechanics: mean cartilage stress, contact area, and contact force of the patellofemoral joint. To compare surgical procedures across various levels of patella distalization, a $2 \times 6$ (procedure $\times$ position) ANOVA with repeated measures was performed. In the case of a significant interaction, separate one-way repeated measures ANOVAs were performed in each surgical procedure. In addition, paired sample t-tests were used to compare between procedures at the each distalization level. All analyses were repeated for cartilage stress, contact area, and contact force.

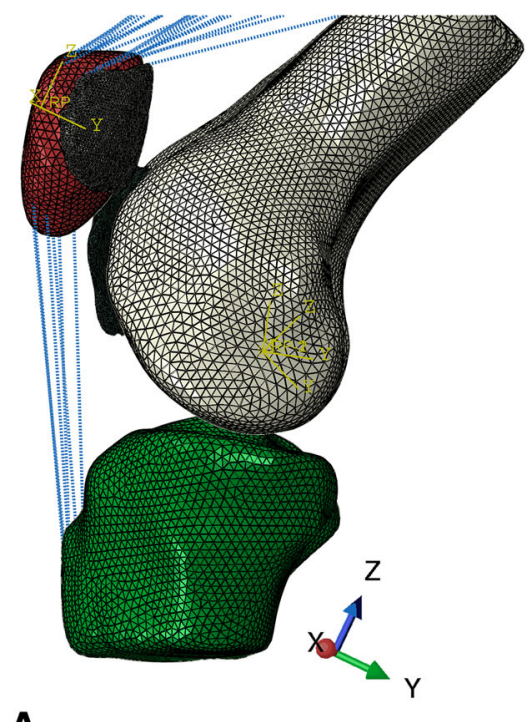

A

Fig. 4A-C (A) The original finite-element model with patella alta, (B) a simulation of the tibial tubercle distalization procedure, and (C) a simulation of the tibial tubercle distalization plus patella tendon tenodesis procedure are shown. Tibial tubercle distalization is simulated by distal transfer of the elements of the tibial tubercle, along with the endpoints of the connectors representing the patella tendon; the patella is distalized accordingly to keep the length of the
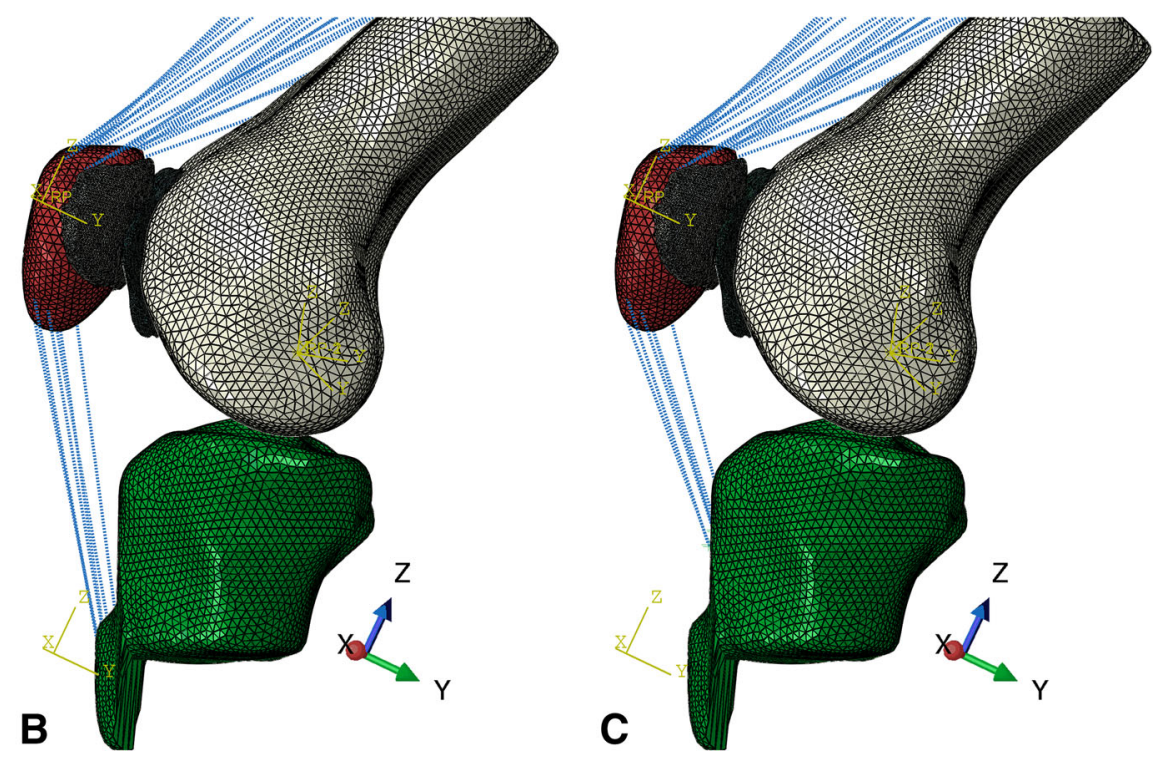

patella tendon unchanged. Patella tendon tenodesis is simulated by attaching the endpoints of the connectors representing the patella tendon back to their original positions. The arrows at the lower right corner and in the illustrations represent the global coordinate system and the local coordinate systems for bone parts, with $\mathrm{X}, \mathrm{Y}$ and $\mathrm{Z}$ axes defined. 


\section{Results}

Biomechanical Comparison Between Distalization and Distalization + Tenodesis

The cartilage stress was found to be lower in the distalization group than the distalization + tenodesis group, at each distance of tibial tubercle distalization (Table 1). Large effect size (Cohen's d) could be observed (mean, 1.60; range, 1.32-1.95). When averaged across distalization distances, distalization and distalization + tenodesis reduced the cartilage stress to $0.91 \pm 0.10 \mathrm{MPa}$ and $0.94 \pm 0.10 \mathrm{MPa}$, respectively, compared with the baseline of $1.02 \pm 0.11 \mathrm{MPa}$.

The contact area was found to be higher in the distalization + tenodesis group than in the distalization group at each distance of tibial tubercle distalization (Table 2). When averaged across distalization distances, distalization and distalization + tenodesis increased the contact area to $279 \pm 46 \mathrm{~mm}^{2}$ and $284 \pm 45 \mathrm{~mm}^{2}$, respectively, compared with the baseline of $268 \pm 39 \mathrm{~mm}^{2}$.

The contact force was found to be lower in the distalization group than in the distalization + tenodesis group at each distance of tibial tubercle distalization (Table 3). When averaged across distalization distances, distalization and distalization + tenodesis reduced the contact force to $430 \pm 94 \mathrm{~N}$ and $456 \pm 92 \mathrm{~N}$, respectively, compared with the baseline of $487 \pm 105 \mathrm{~N}$.

\section{Biomechanical Comparison Among Distalization Distances}

Cartilage stress tended to decline alongside tibial tubercle distalization, but plateaued when the Insall-Salvati ratio approached approximately 0.95 (Fig. 5). For distalization, cartilage stress was lower when the patella was translated distally $4 \mathrm{~mm}, 8 \mathrm{~mm}$, and $12 \mathrm{~mm}$ compared with baseline. Translation of $16 \mathrm{~mm}$ and $20 \mathrm{~mm}$ did not result in a decrease in cartilage stress compared with $12 \mathrm{~mm}$ (Table 4). For distalization + tenodesis, cartilage stress was lower when the tibial tubercle was translated distally $8 \mathrm{~mm}$ compared with baseline. Translation of $12 \mathrm{~mm}, 16 \mathrm{~mm}$, and $20 \mathrm{~mm}$ did not result in decreased cartilage stress compared with $8 \mathrm{~mm}$ (Table 4).

The contact area tended to increase alongside tibial tubercle distalization for distalization + tenodesis, but not for distalization; the trend plateaued when the Insall-Salvati ratio approached approximately 0.95 (Fig. 6). For distalization, the contact area was greater when the tibial tubercle was translated distally $16 \mathrm{~mm}$ compared with baseline. Translating the tibial tubercle to $20 \mathrm{~mm}$ did not result in increased contact area compared with $16 \mathrm{~mm}$ (Table 5).

The contact force tended to decline alongside tibial tubercle distalization (Fig. 7). For distalization, the contact force was lower when the tibial tubercle was translated

Table 1. Cartilage stress (MPa)

\begin{tabular}{|c|c|c|c|c|c|c|}
\hline $\begin{array}{l}\text { Distalization } \\
\text { distances (mm) }\end{array}$ & Distalization & $\begin{array}{l}\text { Distalization }+ \\
\text { tenodesis }\end{array}$ & Mean difference & $\begin{array}{l}95 \% \mathrm{CI} \text { of } \\
\text { difference }\end{array}$ & $\mathrm{p}$ value & Effect size* \\
\hline 0 & $1.02 \pm 0.11$ & $1.02 \pm 0.11$ & & & & \\
\hline 4 & $0.97 \pm 0.10$ & $0.98 \pm 0.10$ & 0.01 & $0.00-0.01$ & 0.001 & 1.49 \\
\hline 8 & $0.93 \pm 0.09$ & $0.95 \pm 0.09$ & 0.02 & $0.01-0.03$ & 0.002 & 1.32 \\
\hline 12 & $0.90 \pm 0.09$ & $0.93 \pm 0.09$ & 0.03 & $0.02-0.04$ & $<0.001$ & 1.56 \\
\hline 16 & $0.88 \pm 0.10$ & $0.92 \pm 0.10$ & 0.04 & $0.02-0.05$ & $<0.001$ & 1.64 \\
\hline 20 & $0.88 \pm 0.11$ & $0.91 \pm 0.11$ & 0.04 & $0.02-0.05$ & $<0.001$ & 1.95 \\
\hline
\end{tabular}

*Cohen's d used.

Table 2. Contact area $\left(\mathrm{mm}^{2}\right)$

\begin{tabular}{lllcrr}
\hline $\begin{array}{l}\text { Distalization } \\
\text { distances }(\mathrm{mm})\end{array}$ & Distalization & $\begin{array}{c}\text { Distalization }+ \\
\text { tenodesis }\end{array}$ & Mean difference & $\begin{array}{c}\text { 95\% CI of } \\
\text { difference }\end{array}$ & p value \\
\hline 0 & $268 \pm 39$ & $268 \pm 39$ & & & Effect size* \\
4 & $273 \pm 37$ & $274 \pm 38$ & 1.7 & $0.4-2.9$ & 0.014 \\
8 & $277 \pm 39$ & $280 \pm 40$ & 2.9 & $1.6-4.2$ & $<0.001$ \\
12 & $280 \pm 43$ & $286 \pm 43$ & 5.7 & $3.1-8.3$ & $<0.001$ \\
16 & $283 \pm 49$ & $290 \pm 49$ & 7.1 & $4.0-10.2$ & $<0.001$ \\
20 & $280 \pm 52$ & $291 \pm 53$ & 10.9 & $5.9-15.9$ & $<0.001$ \\
\hline
\end{tabular}

*Cohen's d used. 
Table 3. Contact force (N)

\begin{tabular}{llcccc}
\hline $\begin{array}{l}\text { Distalization } \\
\text { distances }(\mathrm{mm})\end{array}$ & Distalization & $\begin{array}{c}\text { Distalization } \\
\text { tenodesis }\end{array}$ & Mean difference & $\begin{array}{l}\text { 95\% CI of } \\
\text { difference }\end{array}$ & p value \\
\hline 0 & $487 \pm 105$ & $487 \pm 105$ & & & Effect size* \\
4 & $469 \pm 99$ & $477 \pm 99$ & 8 & $5-11$ & $<0.001$ \\
8 & $450 \pm 93$ & $466 \pm 92$ & 16 & $9.9-23$ & $<0.001$ \\
12 & $430 \pm 88$ & $456 \pm 88$ & 26 & $15-36$ & $<0.001$ \\
16 & $411 \pm 86$ & $446 \pm 87$ & 35 & $20-50$ & $<0.001$ \\
20 & $392 \pm 86$ & $435 \pm 88$ & 43 & $24-63$ & $<0.001$ \\
\hline
\end{tabular}

*Cohen's d used.

Fig. 5 A comparison of cartilage stress between surgery types across various patella translation distances is shown. ${ }^{\circ}$ no significant difference with the minimal value and between values; *significant difference compared with the initial value (0 translation).



Table 4. Pairwise comparison of cartilage stress among distances

\begin{tabular}{|c|c|c|c|c|c|c|c|c|}
\hline \multirow[t]{2}{*}{ Distance } & \multicolumn{4}{|l|}{ Distalization } & \multicolumn{4}{|c|}{ Distalization + tenodesis } \\
\hline & $\begin{array}{l}\text { Mean difference } \\
(\mathrm{MPa})\end{array}$ & $\begin{array}{l}95 \% \mathrm{CI} \text { of } \\
\text { difference (MPa) }\end{array}$ & $\mathrm{p}$ value & Effect size* & $\begin{array}{l}\text { Mean difference } \\
(\mathrm{MPa})\end{array}$ & $\begin{array}{l}\text { 95\% CI of } \\
\text { difference (MPa) }\end{array}$ & $\mathrm{p}$ value & $\begin{array}{l}\text { Effect } \\
\text { size* }\end{array}$ \\
\hline Baseline vs $4 \mathrm{~mm}$ & 0.05 & $0.00-0.10$ & 0.045 & 0.99 & 0.04 & -0.01 to 0.1 & 0.252 & 0.79 \\
\hline Baseline vs $8 \mathrm{~mm}$ & 0.09 & $0.04-0.14$ & $<0.001$ & 1.76 & 0.07 & $0.02-0.12$ & 0.002 & 1.31 \\
\hline Baseline vs $12 \mathrm{~mm}$ & 0.12 & $0.07-0.17$ & $<0.001$ & 2.32 & 0.09 & $0.04-0.14$ & $<0.001$ & 1.67 \\
\hline Baseline vs $16 \mathrm{~mm}$ & 0.14 & $0.09-0.19$ & $<0.001$ & 2.72 & 0.1 & $0.05-0.15$ & $<0.001$ & 1.85 \\
\hline Baseline vs $20 \mathrm{~mm}$ & 0.14 & $0.09-0.19$ & $<0.001$ & 2.8 & 0.1 & $0.05-0.16$ & $<0.001$ & 1.92 \\
\hline $4 \mathrm{~mm}$ vs $8 \mathrm{~mm}$ & 0.04 & -0.01 to 0.09 & 0.279 & 0.77 & 0.03 & -0.03 to 0.08 & 1 & 0.52 \\
\hline $4 \mathrm{~mm}$ vs $12 \mathrm{~mm}$ & 0.07 & $0.02-0.12$ & 0.002 & 1.33 & 0.05 & -0.01 to 0.1 & 0.117 & 0.88 \\
\hline $4 \mathrm{~mm}$ vs $16 \mathrm{~mm}$ & 0.09 & $0.04-0.14$ & $<0.001$ & 1.73 & 0.06 & $0.00-0.11$ & 0.023 & 1.07 \\
\hline $4 \mathrm{~mm}$ vs $20 \mathrm{~mm}$ & 0.09 & $0.04-0.14$ & $<0.001$ & 1.81 & 0.06 & $0.01-0.12$ & 0.012 & 1.14 \\
\hline $8 \mathrm{~mm}$ vs $12 \mathrm{~mm}$ & 0.03 & -0.02 to 0.08 & 1 & 0.55 & 0.02 & -0.03 to 0.07 & 1 & 0.36 \\
\hline $8 \mathrm{~mm}$ vs $16 \mathrm{~mm}$ & 0.05 & $0.00-0.10$ & 0.061 & 0.96 & 0.03 & -0.02 to 0.08 & 1 & 0.55 \\
\hline $8 \mathrm{~mm}$ vs $20 \mathrm{~mm}$ & 0.05 & $0.00-0.10$ & 0.029 & 1.04 & 0.03 & -0.02 to 0.09 & 0.867 & 0.62 \\
\hline $12 \mathrm{~mm}$ vs $16 \mathrm{~mm}$ & 0.02 & -0.03 to 0.07 & 1 & 0.4 & 0.01 & -0.04 to 0.06 & 1 & 0.19 \\
\hline $12 \mathrm{~mm}$ vs $20 \mathrm{~mm}$ & 0.02 & -0.03 to 0.07 & 1 & 0.49 & 0.01 & -0.04 to 0.07 & 1 & 0.26 \\
\hline $16 \mathrm{~mm}$ vs $20 \mathrm{~mm}$ & 0 & -0.05 to 0.05 & 1 & 0.08 & 0 & -0.05 to 0.06 & 1 & 0.07 \\
\hline
\end{tabular}

*Cohen's d used. 
Fig. 6 A comparison of contact area between surgery types across various patella translation distances is shown. ${ }^{\circ}$ no significant difference with the maximal value and between values; *significant difference compared with the initial value (0 translation).



Table 5. Pairwise comparison of contact area among distances

\begin{tabular}{|c|c|c|c|c|c|c|c|c|}
\hline \multirow[t]{2}{*}{ Distance } & \multicolumn{4}{|l|}{ Distalization } & \multicolumn{4}{|c|}{ Distalization + tenodesis } \\
\hline & $\begin{array}{l}\text { Mean difference } \\
\left(\mathrm{mm}^{2}\right)\end{array}$ & $\begin{array}{l}95 \% \mathrm{CI} \text { of } \\
\text { difference }\left(\mathrm{mm}^{2}\right)\end{array}$ & $\mathrm{p}$ value & Effect size* & $\begin{array}{l}\text { Mean difference } \\
\left(\mathrm{mm}^{2}\right)\end{array}$ & $\begin{array}{l}95 \% \mathrm{CI} \text { of } \\
\text { difference }\left(\mathrm{mm}^{2}\right)\end{array}$ & $\mathrm{p}$ value & $\begin{array}{l}\text { Effect } \\
\text { size* }\end{array}$ \\
\hline Baseline vs $4 \mathrm{~mm}$ & -5.1 & -25.8 to 15.5 & 1 & 0.24 & -6.8 & -27.6 to 14.1 & 1 & 0.32 \\
\hline Baseline vs $8 \mathrm{~mm}$ & -9.0 & -29.7 to 11.6 & 1 & 0.43 & -11.9 & -32.8 to 8.9 & 1 & 0.56 \\
\hline Baseline vs $12 \mathrm{~mm}$ & -12.6 & -33.3 to 8.1 & 0.976 & 0.6 & -18.3 & -39.1 to 2.5 & 0.137 & 0.86 \\
\hline Baseline vs $16 \mathrm{~mm}$ & -15.4 & -36.1 to 5.2 & 0.376 & 0.73 & -22.5 & -43.4 to -1.7 & 0.024 & 1.06 \\
\hline Baseline vs $20 \mathrm{~mm}$ & -12.3 & -32.9 to 8.4 & 1 & 0.58 & -23.1 & -43.9 to -2.3 & 0.019 & 1.09 \\
\hline $4 \mathrm{~mm}$ vs $8 \mathrm{~mm}$ & -3.9 & -24.6 to 16.8 & 1 & 0.19 & -5.2 & -26 to 15.7 & 1 & 0.24 \\
\hline $4 \mathrm{~mm}$ vs $12 \mathrm{~mm}$ & -7.5 & -28.1 to 13.2 & 1 & 0.35 & -11.5 & -32.4 to 9.3 & 1 & 0.54 \\
\hline $4 \mathrm{~mm}$ vs $16 \mathrm{~mm}$ & -10.3 & -31.0 to 10.3 & 1 & 0.49 & -15.8 & -36.6 to 5.1 & 0.352 & 0.74 \\
\hline $4 \mathrm{~mm}$ vs $20 \mathrm{~mm}$ & -7.1 & -27.8 to 13.5 & 1 & 0.34 & -16.3 & -37.2 to 4.5 & 0.286 & 0.77 \\
\hline $8 \mathrm{~mm}$ vs $12 \mathrm{~mm}$ & -3.6 & -24.2 to 17.1 & 1 & 0.17 & -6.4 & -27.2 to 14.5 & 1 & 0.3 \\
\hline $8 \mathrm{~mm}$ vs $16 \mathrm{~mm}$ & -6.4 & -27.1 to 14.2 & 1 & 0.3 & -10.6 & -31.4 to 10.2 & 1 & 0.5 \\
\hline $8 \mathrm{~mm}$ vs $20 \mathrm{~mm}$ & -3.2 & -23.9 to 17.4 & 1 & 0.15 & -11.2 & -32 to 9.6 & 1 & 0.53 \\
\hline $12 \mathrm{~mm}$ vs $16 \mathrm{~mm}$ & -2.9 & -23.5 to 17.8 & 1 & 0.14 & -4.2 & -25.1 to 16.6 & 1 & 0.2 \\
\hline $12 \mathrm{~mm}$ vs $20 \mathrm{~mm}$ & 0.3 & -20.3 to 21 & 1 & 0.02 & -4.8 & -25.6 to 16 & 1 & 0.23 \\
\hline $16 \mathrm{~mm}$ vs $20 \mathrm{~mm}$ & 3.2 & -17.5 to 23.8 & 1 & 0.15 & -0.6 & -21.4 to 20.2 & 1 & 0.03 \\
\hline
\end{tabular}

*Cohen's d used.

distally $4 \mathrm{~mm}, 8 \mathrm{~mm}, 12 \mathrm{~mm}$, and $16 \mathrm{~mm}$ compared with baseline; for distalization + tenodesis, the contact force was lower when the tibial tubercle was translated distally $8 \mathrm{~mm}$ compared with baseline (Table 6).

\section{Discussion}

Patella alta has been shown to result in reduced contact area and elevated stress of the patellofemoral joint [30].
Excessive stress exposure has been shown to cause accelerated wear of cartilage, leading to the initiation and progression of osteoarthritis $[4,5]$. Tibial tubercle distalization and tibial tubercle distalization with patella tendon tenodesis are effective procedures in treating patella alta. However, the underlying biomechanical changes associated with these two procedures are not well understood. We investigated the contact mechanics of the patellofemoral joint after the two procedures at various distalization levels using finite-element methods, with the aim of identifying 
Fig. 7 A comparison of contact force between surgery types across various patella translation distances is shown. ${ }^{\circ}$ no significant difference with the minimal value and between values; *significant difference compared with the initial value (0 translation).

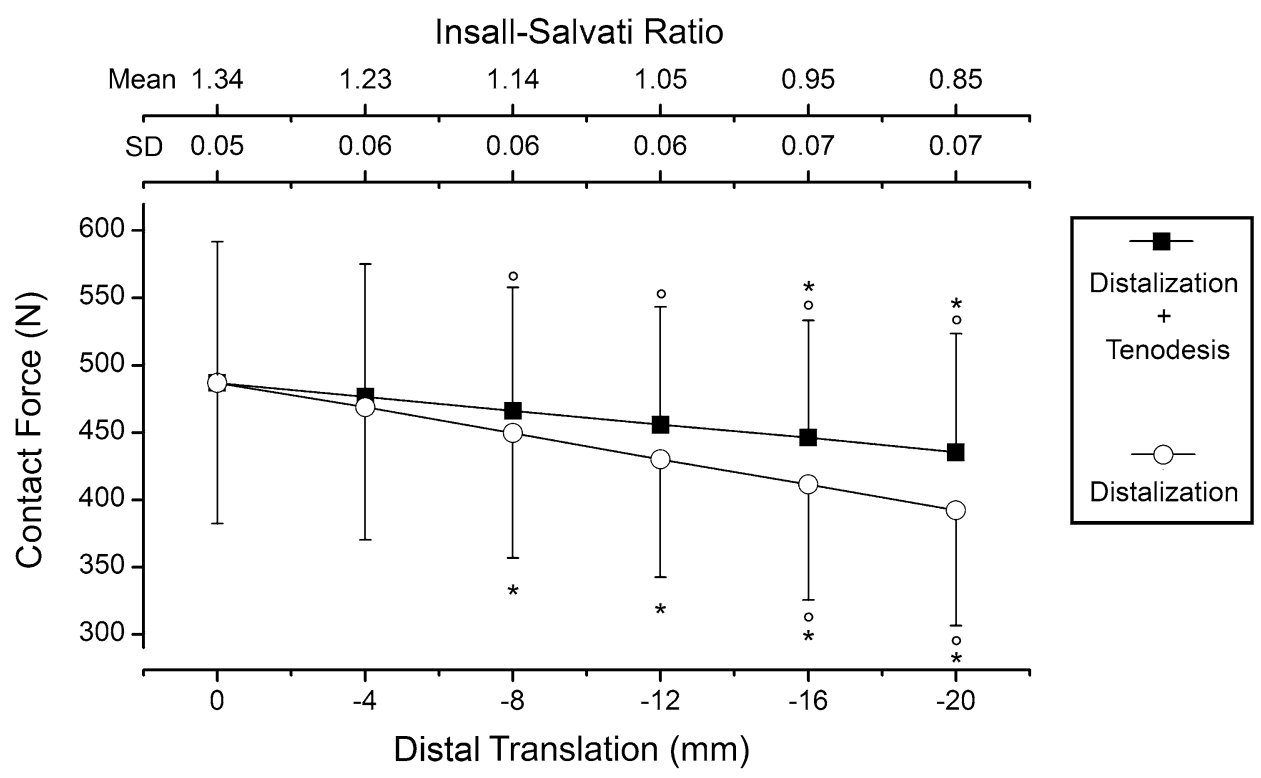

Table 6. Pairwise comparison of contact force among distalization distances

\begin{tabular}{|c|c|c|c|c|c|c|c|c|}
\hline \multirow[t]{2}{*}{ Distance } & \multicolumn{4}{|l|}{ Distalization } & \multicolumn{4}{|c|}{ Distalization + tenodesis } \\
\hline & $\begin{array}{l}\text { Mean difference } \\
(\mathrm{N})\end{array}$ & $\begin{array}{l}95 \% \mathrm{CI} \text { of } \\
\text { difference }(\mathrm{N})\end{array}$ & $\mathrm{p}$ value & Effect size* & $\begin{array}{l}\text { Mean difference } \\
(\mathrm{N})\end{array}$ & $\begin{array}{l}95 \% \mathrm{CI} \text { of } \\
\text { difference }(\mathrm{N})\end{array}$ & $\mathrm{p}$ value & $\begin{array}{l}\text { Effect } \\
\text { size* }\end{array}$ \\
\hline Baseline vs $4 \mathrm{~mm}$ & 18 & -5 to 41 & 0.311 & 0.76 & 10 & -23 to 44 & 1 & 0.3 \\
\hline Baseline vs $8 \mathrm{~mm}$ & 37 & $14-61$ & $<0.001$ & 1.58 & 21 & -12 to 54 & 0.852 & 0.62 \\
\hline Baseline vs $12 \mathrm{~mm}$ & 57 & $34-80$ & $<0.001$ & 2.4 & 31 & -2 to 65 & 0.085 & 0.92 \\
\hline Baseline vs $16 \mathrm{~mm}$ & 76 & $52-99$ & $<0.001$ & 3.18 & 41 & $7-74$ & 0.007 & 1.2 \\
\hline Baseline vs $20 \mathrm{~mm}$ & 95 & $72-118$ & $<0.001$ & 4 & 52 & $18-85$ & 0 & 1.52 \\
\hline $4 \mathrm{~mm}$ vs $8 \mathrm{~mm}$ & 19 & -4 to 43 & 0.194 & 0.82 & 11 & -23 to 44 & 1 & 0.32 \\
\hline $4 \mathrm{~mm}$ vs $12 \mathrm{~mm}$ & 39 & $16-62$ & $<0.001$ & 1.64 & 21 & -12 to 54 & 0.852 & 0.62 \\
\hline $4 \mathrm{~mm}$ vs $16 \mathrm{~mm}$ & 58 & $34-81$ & $<0.001$ & 2.42 & 31 & -3 to 64 & 0.102 & 0.9 \\
\hline $4 \mathrm{~mm}$ vs $20 \mathrm{~mm}$ & 77 & $54-100$ & $<0.001$ & 3.24 & 41 & $8-75$ & 0.006 & 1.21 \\
\hline $8 \mathrm{~mm}$ vs $12 \mathrm{~mm}$ & 20 & -4 to 43 & 0.188 & 0.82 & 10 & -23 to 44 & 1 & 0.3 \\
\hline $8 \mathrm{~mm}$ vs $16 \mathrm{~mm}$ & 38 & $15-61$ & $<0.001$ & 1.6 & 20 & -14 to 53 & 1 & 0.58 \\
\hline $8 \mathrm{~mm}$ vs $20 \mathrm{~mm}$ & 57 & $34-81$ & $<0.001$ & 2.42 & 31 & -3 to 64 & 0.102 & 0.9 \\
\hline $12 \mathrm{~mm}$ vs $16 \mathrm{~mm}$ & 19 & -5 to 42 & 0.262 & 0.78 & 10 & -24 to 43 & 1 & 0.28 \\
\hline $12 \mathrm{~mm}$ vs $20 \mathrm{~mm}$ & 38 & $15-61$ & $<0.001$ & 1.6 & 20 & -13 to 54 & 0.987 & 0.6 \\
\hline $16 \mathrm{~mm}$ vs $20 \mathrm{~mm}$ & 19 & -4 to 43 & 0.197 & 0.82 & 11 & -23 to 44 & 1 & 0.32 \\
\hline
\end{tabular}

*Cohen's d used.

the better procedure and the ideal distalization level. We found that tibial tubercle distalization led to lower cartilage stress than distalization + tenodesis. The distalization level that reduced the Insall-Salvati ratio to approximately 0.95 resulted in the lowest cartilage stress, which could not be decreased further by additional distalization.

As with any computation study related to clinical practice, there are important limitations that need to be considered when interpreting the results. First, the biomechanical parameters were quantified at only $45^{\circ}$ knee flexion. As such, contact mechanics at larger or smaller knee flexion angles cannot be directly inferred from this study. Nevertheless, we speculate that distalization may still have advantages over distalization + tenodesis at other knee flexion angles in terms of contact stresses, as the anatomic features associated with the two procedures can be maintained. Future studies using dynamic finite-element models which can quantify cartilage stresses across a wide range of knee flexion angles may be able to overcome this limitation. Second, we simplified the finite-element models 
by constraining patella rotation in the transverse and coronal planes, which substituted for the function of peripatellar soft tissue. A more-advanced model with complete soft tissue simulation would likely yield results that would be more generalizable clinically. Third, the potential changes in patella tendon stiffness and passive quadriceps muscle tension were not considered after tenodesis. However, given the relatively high stiffness of the patella tendon and relatively low level of quadriceps muscle loading used in this study, we speculate that our findings would not have been affected by these methodologic assumptions.

Additional limitations include the use finite-element models created from a relatively small number of females with and without patellofemoral pain using a single criterion (Insall-Salvati ratio $>1.2$ ). Other anatomic characteristics that may have affected patellofemoral stability were not specifically considered in our selection criteria. As such, the generalizability of our results may be limited. Additionally, we compared only direct distalization procedures, however, medialization of the tibial tubercle also is used frequently in treating patella alta. Finally, the results of our study were based on computational simulations and thus remain at a theoretical level. Nonetheless, our findings provide empirical data to support the development of clinical studies related to both procedures. Clinical trials with patient followup will be necessary before any conclusions can be applied to practice.

A primary finding of our study was that both procedures reduced patellofemoral joint stress compared with the initial model condition. However, distalization can lead to lower cartilage stress than distalization + tenodesis at each distalization level. In addition, the former needed less distalization distance to achieve a lower stress level in relation to the baseline. A possible explanation for this result is the more-distal insertion of patella tendon. The lower insertion resulted in an increased moment arm of the patella tendon about the knee axis of rotation, thus reducing the quadriceps muscle force and patella tendon force required to generate the required knee moment (which was held constant throughout the study). In addition, the lower tendon insertion associated with distalization resulted in a more-vertical line of pull of the patella tendon compared with distalization + tenodesis, thus effectively reducing the resultant (compressive) force of the quadriceps and patella tendon force vectors. This is in agreement with results reported by Ahmad et al. [1] of an experimental study which tested the effects of different patella tendon length and insertion on patellofemoral biomechanics.

The benefits of distalization have been well documented in previous clinical studies [2, 22, 23]. Poor patellofemoral anatomy, such as lateral tilt of patella can be improved substantially after surgery [22, 23]; patient-reported outcomes such as the Lysholm and Kujala scores also could be substantially improved [2, 22, 23]. Distalization + tenodesis also showed ideal clinical outcomes at long-term followup, in objective and subjective assessments [20]. According to the results of the current study, distalization might be the more favorable of the two procedures, as it not only reduced cartilage stress more effectively, but also saved an extra step of patella tendon tenodesis, which could help reduce the time and cost of the surgery. Nevertheless, there may be extra benefits associated with use of the additional tenodesis for the treatment of patella alta. For example, patella alta has been shown to result from an elongated patella tendon as opposed to a more-proximal patella tendon insertion [21]. It has been suggested that an elongated patella tendon may be a risk factor for patella instability in the coronal plane $[20,21]$. Thus, shortening of the patella tendon may provide additional benefits beyond relief of cartilage stress. Future long-term clinical studies of the two procedures, doing anatomic measurements, and obtaining patient-reported scores would provide more and stronger evidence.

Our model suggested that the favorable level to distalize the tubercle was to restore the Insall-Salvati ratio to approximately 0.95 . A distalization of $4 \mathrm{~mm}$ decreased the Insall-Salvati ratio by 0.1 , therefore patient-specific distalization distance may be quantified based on preoperative measurements. The relationship between patellofemoral contact mechanics and patella height have been investigated. Ward and Powers [30] reported that a high-riding patella led to increased patellofemoral stress during walking and running compared with patellas from healthy controls. Similar results were reported by Luyckx et al. [17] for simulated knee bending. On the contrary, the patellofemoral joint could benefit with lowering the patella height from high, as seen in our study. However, excessive distalization of the tibial tubercle could cause patella infera, which did not seem to have additional benefits. Using mathematical calculation [29] and cadaver tests [26], these studies suggested that a low-lying patella could result in lower patellofemoral contact forces compared with normal, which corresponded to results in our study. In addition, we found that cartilage stress plateaued when patella height had been corrected close to normal (InsallSalvati ratio approximately 1.0); further distalization did not provide lower values. Clinically, a low-lying patella or elevated tibiofemoral joint line also may lead to several complications such as joint stiffness, decreased ROM, and anterior knee pain $[11,14,28]$. Therefore, we advocate proper distalization of the tibial tubercle to correct the Insall-Salvati ratio to normal. Overdistalization is not suggested. 
Distalization and distalization + tenodesis can effectively improve patellofemoral joint contact mechanics. However, this finite-element analysis suggests that lower cartilage stresses can be achieved using distalization. An Insall-Salvati ratio of 0.95 appears to be an ideal level of distalization. Our study was based on model simulation, therefore the results should be verified by future clinical studies which will provide more and stronger evidence for surgeons to translate the conclusions into clinical practice.

\section{References}

1. Ahmad CS, Kwak SD, Ateshian GA, Warden WH, Steadman JR, Mow VC. Effects of patellar tendon adhesion to the anterior tibia on knee mechanics. Am J Sports Med. 1998;26:715-724.

2. Al-Sayyad MJ, Cameron JC. Functional outcome after tibial tubercle transfer for the painful patella alta. Clin Orthop Relat Res. 2002;396:152-162.

3. Besier TF, Gold GE, Delp SL, Fredericson M, Beaupré GS. The influence of femoral internal and external rotation on cartilage stresses within the patellofemoral joint. J Orthop Res. 2008;26: 1627-1635.

4. Carter DR, Beaupré GS, Wong M, Smith RL, Andriacchi TP, Schurman DJ. The mechanobiology of articular cartilage development and degeneration. Clin Orthop Relat Res. 2004;427 (suppl):S69-77.

5. Cicuttini FM, Forbes A, Yuanyuan W, Rush G, Stuckey SL. Rate of knee cartilage loss after partial meniscectomy. J Rheumatol. 2002;29:1954-1956.

6. Elias JJ, Bratton DR, Weinstein DM, Cosgarea AJ. Comparing two estimations of the quadriceps force distribution for use during patellofemoral simulation. J Biomech. 2006;39:865-872.

7. Elias JJ, Carrino JA, Saranathan A, Guseila LM, Tanaka MJ, Cosgarea AJ. Variations in kinematics and function following patellar stabilization including tibial tuberosity realignment. Knee Surg Sports Traumatol Arthrosc. 2014;22:2350-2356.

8. Elias JJ, Saranathan A. Discrete element analysis for characterizing the patellofemoral pressure distribution: model evaluation. $J$ Biomech Eng. 2013;135:81011.

9. Farrokhi S, Keyak JH, Powers CM. Individuals with patellofemoral pain exhibit greater patellofemoral joint stress: a finite element analysis study. Osteoarthr Cartilage. 2011;19:287-294.

10. Grelsamer RP, Meadows S. The modified Insall-Salvati ratio for assessment of patellar height. Clin Orthop Relat Res. 1992;282: 170-176.

11. Guido W, Christian H, Elmar H, Elisabeth A, Christian F. Treatment of patella baja by a modified Z-plasty. Knee Surg Sports Traumatol Arthrosc. 2015 Mar 19. [Epub ahead of print]

12. Ho KY, Keyak JH, Powers CM. Comparison of patella bone strain between females with and without patellofemoral pain: a finite element analysis study. J Biomech. 2014;47:230-236.

13. Kannus PA. Long patellar tendon: radiographic sign of patellofemoral pain syndrome: a prospective study. Radiology. 1992;185:859-863.

14. Kazemi SM, Daftari Besheli L, Eajazi A, Miniator Sajadi MR, Okhovatpoor MA, Farhang Zanganeh R, Minaei R. Pseudopatella baja after total knee arthroplasty. Med Sci Monit. 2011;17:CR292-296.

15. Kujala UM, Österman K, Kvist M, Aalto T, Friberg O. Factors predisposing to patellar chondropathy and patellar apicitis in athletes. Int Orthop. 1986;10:195-200.
16. Lee PP, Chalian M, Carrino JA, Eng J, Chhabra A. Multimodality correlations of patellar height measurement on X-ray, CT, and MRI. Skeletal Radiol. 2012;41:1309-1314.

17. Luyckx T, Didden K, Vandenneucker H, Labey L, Innocenti B, Bellemans J. Is there a biomechanical explanation for anterior knee pain in patients with patella alta?: influence of patellar height on patellofemoral contact force, contact area and contact pressure. J Bone Joint Surg Br. 2009;91:344-350.

18. Magnussen RA, De Simone V, Lustig S, Neyret P, Flanigan DC. Treatment of patella alta in patients with episodic patellar dislocation: a systematic review. Knee Surg Sports Traumatol Arthrosc. 2014;22:2545-2550.

19. Marteau E, Burdin P, Brilhault JM. Gracilis tendon transfer associated with distal alignment for patella alta with recurrent dislocations: an original surgical technique. Orthop Traumatol Surg Res. 2011;97(4 suppl):S5-11.

20. Mayer C, Magnussen RA, Servien E, Demey G, Jacobi M, Neyret $\mathrm{P}$, Lustig S. Patellar tendon tenodesis in association with tibial tubercle distalization for the treatment of episodic patellar dislocation with patella alta. Am J Sports Med. 2012;40:346-351.

21. Neyret P, Robinson A, Le Coultre B, Lapra C, Chambat P. Patellar tendon length: the factor in patellar instability? Knee. 2002;9:3-6.

22. Otsuki S, Nakajima M, Fujiwara K, Okamoto Y, Iida G, Murakami T, Neo M. Influence of age on clinical outcomes of threedimensional transfer of the tibial tuberosity for patellar instability with patella alta. Knee Surg Sports Traumatol Arthrosc. 2016 Feb 2. [Epub ahead of print]

23. Otsuki S, Nakajima M, Oda S, Hoshiyama Y, Fujiwara K, Jotoku T, Neo M. Three-dimensional transfer of the tibial tuberosity for patellar instability with patella alta. J Orthop Sci. 2013;18:437442.

24. Pritsch T, Haim A, Arbel R, Snir N, Shasha N, Dekel S. Tailored tibial tubercle transfer for patellofemoral malalignment: analysis of clinical outcomes. Knee Surg Sports Traumatol Arthrosc. 2007;15:994-1002.

25. Shah KS, Saranathan A, Koya B, Elias JJ. Finite element analysis to characterize how varying patellar loading influences pressure applied to cartilage: model evaluation. Comput Methods Biomech Biomed Engin. 2015;18:1509-1515.

26. Singerman R, Davy DR, Goldberg VM. Effects of patella alta and patella infera on patellofemoral contact forces. J Biomech. 1994;27:1059-1065.

27. Stefanik JJ, Zhu Y, Zumwalt AC, Gross KD, Clancy M, Lynch JA, Frey Law LA, Lewis CE, Roemer FW, Powers CM, Guermazi A, Felson T, Felson DT. Association between patella alta and the prevalence and worsening of structural features of patellofemoral joint osteoarthritis: the multicenter osteoarthritis study. Arthritis Care Res. 2010;62:1258-1265.

28. Tecklenburg K, Dejour D, Hoser C, Fink C. Bony and cartilaginous anatomy of the patellofemoral joint. Knee Surg Sports Traumatol Arthrosc. 2006;14:235-240.

29. Van Eijden TM, Kouwenhoven E, Weijs WA. Mechanics of the patellar articulation: effects of patellar ligament length studied with a mathematical model. Acta Orthop Scand. 1987;58:560566.

30. Ward SR, Powers CM. The influence of patella alta on patellofemoral joint stress during normal and fast walking. Clin Biomech (Bristol, Avon). 2004;19:1040-1047.

31. Ward SR, Terk MR, Powers CM. Patella alta: association with patellofemoral alignment and changes in contact area during weight-bearing. J Bone Joint Surg Am. 2007;89:1749-1755.

32. Yamaguchi GT, Zajac FE. A planar model of the knee joint to characterize the knee extensor mechanism. $J$ Biomech. 1989;22:1-10. 